\title{
LA PRUEBA ANTICIPADA COMO HERRAMIENTA PARA REDUCIR LA CULTURA DE LITIGIO RECURRIENDO AL DERECHO COMPARADO
}

\author{
Emerson Noe Guevara Reyes ${ }^{1}$ \\ Wilfredo Guevara Trujillo ${ }^{2}$
}

DOI: https://doi.org/10.5377//rd.v41i1.10502

\section{RESUMEN:}

El siguiente artículo está desarrollado con la noción de ofrecer posibles soluciones para reducir la cultura de litigios y que las partes puedan tener mecanismos para poder llegar a acuerdos consensuados y resolver el conflicto, todo esto desde elementos que la misma normativa jurídica tipifica, para ello planteo un breve pero necesario análisis en derecho comparado para demostrar cual es la noción, que el mundo está teniendo en cuanto a la relevancia de las partes para resolver sus conflictos, asimismo ofrezco el mecanismo de la prueba anticipada como una nueva noción, con características de autonomía con la mera intención de información, influenciada principalmente por el derecho brasileño procesal vigente y de origen de Common Law.

\section{PALABRAS CLAVE:}

Cultura de Litigio, Prueba Anticipada, Derecho a Prueba, Derecho Comparado. Derecho Autónomo a Prueba.

Fecha de recepción: 31 de agosto de 2020 Fecha de aprobación:23 de noviembre de 2020

1 Abogado y master em derecho Procesal Egresado de la Universidade Federal de Espirito Santo de la Republica de Brasil. Correo Electrónico: maidennoe@hotmail.com

2 Abogado y Notário egresado de la Universidad Nacional Autonoma de Honduras.

Correo Electrónico: wilfredo2015guevara@gmail.com 


\title{
ANTICIPATED PROOF TO REDUCE THE CULTURE OF LITIGATION USING COMPARATIVE LAW
}

\section{Emerson Noe Guevara Reyes ${ }^{3}$ Wilfredo Guevara Trujillo ${ }^{4}$}

\section{DOI: https://doi.org/10.5377/Ird.v41i1.10502}

\section{ABSTRACT:}

The following article is developed with the notion of offering possible solutions to reduce the litigation culture and that the parties may have mechanisms to reach consensual agreements and resolve the conflict, all this from elements that the same legal regulations typify, for this I propose a brief but necessary analysis in comparative law to demonstrate what is the notion, that the world is having in terms of the relevance of the parties to resolve their conflicts, I also offer the mechanism of advance evidence as a new notion, with characteristics of autonomy with the mere intention of information, mainly influenced by the current Brazilian procedural law and Common Law origin.
\end{abstract}

\section{KEY WORDS:}

Litigation Culture, Advance Evidence, Right to Proof, Comparative Law. Autonomous Right to Trial

Date received: August 31, 2020 Approval date: November 23, 2020

3 Lawyer and Master in Procedural Law Graduated from the Federal University of Espirito Santo of the Republic of Brazil. Email: maidennoe@hotmail.com 4 Lawyer and Notary Public graduated from the Universidad Nacional Autonoma de Honduras.

Email: wilfredo2015guevara@gmail.com 


\section{INTRODUCCIÓN}

"El arte del proceso no es esencialmente otra cosa que el arte de administrar las pruebas" (Bentham, 1971, p10.).

Este trabajo pretende ofrecer algunos criterios, para intentar controlar o reducir esa clara y manifiesta cultura de litigio que tenemos en Honduras, con ello me refiero al alto impacto peyorativo que esta cultura tiene para la solución de conflictos o composición de la Litis.

El mundo ya percibió que la cultura de Litigio $^{5}$ es un problema que perjudica en una de sus facetas al acceso a la Justicia, en cuanto a plazo razonables nos referimos, recordando que Justicia tardía no es justicia, o a manera de ejemplo aquella cultura que en Honduras, de presentar un recurso judicial, que la misma norma jurídica expresa un plazo de días para su resolución, y en la realidad este es resuelto en dos años en uno de los mejores casos bajo el argumento de "mora Judicial", aspecto que dicho sea de paso ya es considerado y tomado tanto por los jueces y abogados litigantes y ciudadanía con toda la normalidad.

Esto sin duda moldea un escenario de conformidad, misma que es lesiva a una sociedad bajo un estado de derecho democrático constitucional, al tenor que la anhelada Justicia se ve vulnerable por nuestro tipo de actuar y peor todavía por nuestro silencio e indiferencia hacia ello.

5 Entendiendo esta como un enfrentamiento de posiciones antagónicas sobre alguna relación jurídica dentro de un órgano jurisdiccional.
Mi propuesta, es estudiar el derecho de proceso para evitar los procesos judiciales, y así no solo garantizar la justicia si no ofrecer un oxígeno a los órganos jurisdiccionales que la sociedad y sus conflictos sean resueltos por ellos mismos y no necesaria mente por el poder judicial como manera principal o única solución.

Esto pretendo plantearlo estableciendo la importancia del derecho a la información y el derecho a prueba, considerando que cuando en un conflicto las partes tienen información sobre algún asunto, prefieren o puede optar a tener posiciones diferentes y solucionar sus problemas con acuerdos extrajudiciales.

Para ello ofrezco unas figuras jurídicas como ejemplo de acceso a información, denominada como la prueba autónoma anticipada, que voy a desarrollar en las premisas a lo largo de trabajo.

\section{METODOLOGÍA}

El método comparativo en cuanto se habla de actividad de cognición es parte inherente al proceso de construcción del conocimiento en todas las áreas de la ciencia social (Schneider. Job.1998, p, 49.) por ello el camino seleccionado para desarrollar el presente artículo, es el método comparativo y método descriptivo, debido a la ardua necesidad de observación sensorial y análisis de la comparabilidad de figuras jurídicas dentro de contextos jurídicos, en base a estos métodos es como pretendo obtener tener las cualidades necesarias que sean arrojadas dentro de un resultado que sea objetivo, con la finalidad de generar, sugestiones, criticas, y propuestas. 
Por su naturaleza analizan relaciones de factores principalmente entre fenómenos, con semejanzas por su naturaleza, conservando sus fieles características distintas por sus diversas causas, es decir, con escenarios distintos de desarrollo tiene como herramienta principal y fundamental ese punto analítico orientado a la comparación y descubrimiento el cual arroja un resultado, con las convergencias de teorías que serán el acápite y centralización de los fundamentos empleados en la investigación, la variabilidad y la complejidad que el proceso comparativo contrae por sí mismo a través del énfasis de los posibles aportes jurídicos que puedan ser expuestos.

Pretendo hacer una inmersión tangible del campo fenoménico de estudio, así como desarrollar y establecer de forma clara los temas emergentes que giran en torno al derecho procesal o más específicamente al derecho probatorio y la institución de la Prueba anticipada como propuesta, por lo que la generación de conocimiento a través de la compatibilidad de semejanzas y diferencias con el destino de iluminar aspectos desconocidos a través de aportes que viene siendo discutida por la doctrina, para aportar un grano en desarrollo de conocimiento a la academia jurídica.

El presente trabajo es el resultado de una de las varias conclusiones de la tesis del mestrado presentada y defendida en la Universidad Federal do Espirito Santo en la República Federal del Brasil, en el Programa de Direito Processual, para obtener el grado de Master en derecho procesal por unos de los autores del presente artículo.

\section{CULTURA DE LITIGIO}

Actualmente es innegable que Honduras vive dentro de una Cultura de litigio, de hecho no existe ninguna cultura en el mundo que no sea objeto de litigio es decir el surgimiento de la Litis, pero que si se percibe en mayor o menor medida su impacto dentro de la sociedad, que son instaurado dentro de un órgano judicial, muchos casos perfectamente pueden ser resueltos de otra forma. Ejemplo de ello son los métodos alternos de solución de conflicto, como la mediación o el arbitraje sin embargo estos tienen aspectos positivos como negativos que por la naturaleza de este artículo no desarrollare.

De esta forma, tal y como el Doctor Chase, (2014) manifesta " Qualquier modo de Solucion de litígios aprobado por las sociedades es el resultado de las decisiones consientes e inconscientes hechas em los limites del conocimiento, de las creencias y de la estrutura social disponibles." (p.20). (traduccion libre)

El punto central es esta cultura de litigio que nuestra sociedad atañe en equivocarse, en cuanto el método adecuado de solucionar el conflicto, es decir donde existe cambio de bienes, prestaciones de servicios celebraciones de contratos etc. Es inevitable que dentro de una sociedad existan conflictos entre partes derivados de la relación jurídica en sus respectivos casos.

La cuestión es que nuestra misma nube de solución de conflictos nos orienta a una sola puerta que es el sistema judicial y no a un sistema de multipuertas como son los 
métodos alternos de solución de conflictos, es menester mencionar que estos métodos existen y son reconocidos por la ley hondureña sin embargo no es la primer opción que tiene los hondureños para dirimir sus controversias.

Actualmente esa puerta como es el Sistema Judicial, representado por la Corte Suprema de Justicia y todos sus órganos jurisdiccionales, esta abismalmente saturado de procesos judiciales, según la corte suprema de justicia esta cuenta con 185,000.00 casos en mora judicial desde Julio del 2016, que han ido formando parte de la cosa juzgada y en Diciembre 2018 llega a la cantidad de 71,036 expedientes aun en mora judicial.

Este problema en definitiva, si atañe a la corte suprema de Justicia sin embargo no es la única responsable, si no es por nuestra cultura litigiosa, y es ahí donde tenemos que mejorar, pensar y actuar. Asimismo quiero dejar claro que con esto no quiero que se me mal interprete, no estoy tratando de decir que la responsabilidad de la corte debe ser menos o que está saturada por culpa solamente de la sociedad, esto es sin duda un resultado que se ve reflejado en considerables negligencias estatales en la administración de la justicia y por supuesto por el gobierno central que le compete en cada época histórica hondureña, solo ese tema daría para realizar otro trabajo de investigación.

Actualmente la corte suprema de justicia cuenta con planes para la erradicación de la mora judicial, sin embargo la mayor parte de ellas están orientadas a controlar la mora dentro de los diferentes órganos jurisdiccionales y no antes de que estos problemas lleguen a formar parte de la mora judicial ${ }^{6}$.

Es decir la Corte suprema de Justicia no está enfocándose en la prevención de la mora judicial si no que está enfocándose en el tratamiento de esta cuando ya está dentro de los órganos jurisdiccionales, en otras palabras no se está previendo o anticipando al problema si no que solamente se está intentando lidiar con él.

Esta cultura de Litigio no solo atañe a la sociedad en general sino que también hasta los mismos abogados, que enfrentado esta situación con características de pensar que su labor o su buena gestión como abogado radica en la solución de un problema a través del poder judicial y más todavía aquella cultura de litigiosidad en cuanto a presentar recursos solamente para demostrar a su cliente "que se hizo lo que se pudo" para solventar la causa por la que fue contratado, inclusive a sabiendas que dicho recurso judicial no iba tener ni el más mínimo porcentaje de éxito, o como mejor expresa e informa el poder judicial a través del plan nacional de erradicación de la mora judicial:

Si bien, hemos dicho y reconocemos que en algún porcentaje la mora judicial obedece a causas atribuibles al Poder Judicial, no menos cierto es que en muchas ocasiones dichas causas escapan de la decisión del Juez o Magistrado, concurriendo, en consecuencia, como motivos de la mora judicial, conductas de las partes y/o de otros intervinientes en los procesos jurisdiccionales, que van desde las llamadas tácticas dilatorias, hasta la ausencia de trámite o inactividad en aquellos casos que

6 Para mayor Ilustración ver: http://www.poderjudicial. gob.hn/PNEMJ/Paginas/default.aspx. Ultimo Acceso en: 22/7/2020. 
no pueden impulsarse oficiosamente $\mathrm{y}$, por ende, existe la obligación de instarlos. (Corte Suprema de Justicia de Honduras, 2006, p.10).

Esta cultura es materializada desde la sociedad y principalmente en las aulas de formación universitaria en el caso de los abogados, en donde al estudiante se forma bajo la noción que iniciar un proceso judicial o presentar recursos judiciales o tácticas dilatorias independientemente de su retraso es para una supuesta lucha, o como popularmente se conoce para darle vuelta a un asunto. Pero no se enseña cual es el verdadero propósito del mismo, desde puntos morales y éticos y que en definitiva no se trata de Demostrarle al cliente que se luchó por ganar la causa por la que fue contratado. Además del cliente estar satisfecho por el intento del abogado pensando que esa fue la mejor forma por aferrarse y no por tratar de mejor manera el conflicto.

Es por ello que el romper con este paradigma no solo es un problema que atañe a los abogados, es una situación que estamos obligados a resolver como miembros de una sociedad y como abogados en su respectivos casos por lo que el papel de los tribunales, universidades y estudiantes de derecho y abogados litigantes, es siempre tratar adecuadamente el conflicto y concluir cuál de todos los sistema para resolver la colisión de intereses es mejor para la composición de la Litis incluyendo los acuerdos extrajudiciales.

\section{INFORMACIÓN COMO ERRAMIENTA PARA SOLUCIONAR CONFLICTOS}

Una de los criterios que quiero proponer en este artículo para el mejor tratamiento del conflicto y la solución a través de la autocomposición entre las partes es ofrecer herramientas jurídicas que brinden información sobre el caso, al tenor que es evidente que con pruebas, con datos, con información pre existente de un proceso, las posibilidades de conciliar o mediar o negociar el conflicto pueden verse potenciados.

Es por ello que quiero desarrollar la experiencia en el derecho anglosajón para que podamos entender con una mejor visión el poder del acuerdo pre judicial a raíz de la información como herramienta para solucionar el conflicto y de esta manera plantear en base a la realidad hondureña elementos que podrían aplicarse.

\section{DISCLOSURE (DERECHO INGLES)}

Manifestado a grandes rasgos los criterios del porque Honduras está hundida en una cultura de litigio, procedo a la línea de estudiar el derecho de proceso para evitar procesos a través del derecho comparado.

En este acápite iniciare con el derecho anglosajón inglés y el derecho de Estados unidos de América, específicamente en las figuras denominadas como Discovery y el disclousure.

En el derecho ingles el disclousure (revelación) principalmente en los criterios denominados como pre action o entenderlo mejor como una etapa antes de la declaración de la demanda, es decir un proceso pre judicial, así como la fase pre trial entendiéndola como la fase anterior a la audiencia de juzgamiento regulada principalmente por el artículo 31.2 de 
las Civil prodecure Rules, bajo la modalidad de los pre action se materializa mediante los pre- action protocols que se resumen, en que forma las partes antes de iniciar un proceso pueden intercambiar información en relación al conflicto, por lo que se conectan con las finalidades incluidas la autocomposición del conflicto, esta información evidentemente cubre el ámbito probatorio, por lo que se intercambian pruebas como suporte cognoscitivo de información para tanto discutir o formar la convicción en iniciar un proceso, verificar los chances de ganar o perder, o simplemente para llegar a una solución consensuada del conflicto (Laux, 2015, p.5)

Es menester mencionar que en la Jurisdicción inglesa la producción de pruebas en estas etapas han alcanzado el 60\% soluciones auto compositivas en que los pre trial protocolos fueron utilizados dejando en evidencia como está siendo entendido las múltiples puertas para que las partes puedan tener acceso a medios de autocomposición y que la misma ley ofrece a sabiendas de que el conflicto nunca ira a terminar como fenómeno dentro de una sociedad. (Silveira, 2010, p.52)

\section{DISCOVERY. ( DERECHO DE ESTADOS UNIDOS DE AMÉRICA)}

Asimismo el denominado Discovery (Estados Unidos de América) consiste en una línea jurídica similar, es decir, otorga una alta participación a las partes antes de un proceso en donde se intercambia información que puede promover sin duda la resolución del conflicto entre las partes, inclusive se puede citar a un tercero para que brinde información necesaria para solucionar el conflicto todo esto sin la ayuda o dirección del poder judicial esto regulado principalmente por la Federal Rules of Civil prodecure de 1938 regla 26(b)(1).

En sentido al revelarse la información central del conflicto ya existe un panorama mayormente capaz de ofrecer elementos deductivos necesarios para que las partes puedan tomar una decisión sea esta presentar la acción futura procesal o simplemente solucionar su controversia mediante un acuerdo.

\section{ACCIÓN AUTÓNOMA DE LA PRUEBA ANTICIPADA EN EL DERECHO BRASILEÑO BAJO LA REGULACIÓN DEL CÓDIGO DE PROCESO CIVIL DEL 2015 COMO MODELO EN EL CIVIL LAW LATINOAMERICANO}

Tanto las figuras del disclousure y el Discovery son figuras eminente del derecho anglosajón o del denominado common Law, por ello y como prueba de que el mundo está empezando a entender que la norma jurídica debe de ofrecer todo los criterios necesarios para que las partes puedan solventar su conflicto entre ellas, y que no necesariamente en algunos tipos de conflictos se debe de presentar acciones ante el poder judicial, traigo como ejemplo más cercano a nuestra realidad jurídica hondureña de orden civil law y latinoamericano como es el derecho brasileño.

En el derecho brasileño recientemente entró en vigencia el código de proceso civil 
del $2015^{78}$ que trae consigo un sinnúmero de institutos jurídicos de suma relevancia, incluyendo innovaciones en el ámbito del derecho probatorio, en este sentido se encuentra específicamente la acción autónoma de prueba anticipada que desarrollare a continuación.

El CPC/2015, tipifica un conjunto de normas jurídicas que intentan promover un procedimiento expedito y practico que garantice la tutela adecuada de los derechos, con la finalidad de la pacificación de conflictos originados por la sociedad, un aspecto evidente de ello es el otorgamiento de tres artículos, que se encuentran específicamente del artículo 381 al 383, como delimitador del Capítulo de las pruebas, sección II de la producción de la prueba anticipada del compendio legal aludido, y no en las figuras cautelares que el CPC/2015 contempla, por lo que se puede inferir que el legislador de este país nunca tuvo intención de establecer a la figura de la prueba anticipada, con carácter de urgencia o cautelar (Nery Junior y Andrade, 2018. p.381) por lo menos no principalmente como si es en el Código de proceso civil de Honduras del 2007 como se verá adelante.

Las hipótesis principales donde cabe el derecho autónomo de producción de prueba anticipada son reguladas literalmente por el artículo 381 del CPC/2015.

\footnotetext{
7 Todos los artículos expresados literalmente son obtenidos de la obra BRASIL. Ley n ${ }^{\circ}$ 13.105, de 16 de Março de 2015. CPC brasileiro traduzido para a língua espanhola: código de proceso civil brasileño de 2015. ARRUDA, Teresa Alvim. DIDIER JUNIOR, Fredie. Tradução CAVANI, RENZO. Salvador: Jus Pudivm.

8 Em adelante $\mathrm{CPC} / 2015$

9 Art. $381^{\circ}$. La producción anticipada de prueba será admitida en los casos en que: I - exista fundado temor de que se vuelva
}

Como se puede percibir, una de las innovaciones es la desvinculación del requisito de peligro o de Urgencia en cuanto a la producción autónoma de la prueba (Yarshell, 2015.p.1027.) El artículo 381 inciso I, solo necesita que exista un miedo infundado de que la fuente del medio probatorio no pueda practicarse en un futuro, por lo que ratifica aún más su carácter autónomo a un proceso principal o a un plazo imperativo (Sampaio, 2019, p.118). en esta misma lógica en cuanto a la no dependencia de un proceso principal como fundamental requisito para la admisión de la producción autónoma de la prueba fundamentado en el derecho de acción, y, en consonancia con el inciso II del artículo citado, el código en base a la idea democrática, y de fomento de autocomposición, otorga la viabilidad para que las partes puedan producir pruebas anticipadas con la idea esencial de corroborar sus chanches de un posible futuro proceso y más explícitamente, si la producción de esta prueba tiene un fundamento eminentemente para provocar una autocomposición entre las partes. Tal y

imposible o muy difícil la verificación de ciertos hechos en la pendencia de la acción; II - la prueba a ser producida sea susceptible de viabilizar la autocomposición u otro medio adecuado de solución de conflicto; III - el previo conocimiento de los hechos pueda justificar o evitar la proposición de la acción. $\$ 1^{\circ}$. El listado de bienes observará lo dispuesto en esta Sección cuando tenga por finalidad solamente la realización de documentación y no la práctica de actos de aprehensión. $\S 2^{\circ}$. La producción anticipada de prueba es de competencia del juzgado del foro donde esta deba ser producida o del foro del domicilio del demandado. $\$ 3^{\circ}$. La producción anticipada de prueba no previene la competencia del juzgado para la acción que vaya a ser propuesta. $\$ 4^{\circ}$. El juzgado estadual tiene competencia para la producción anticipada de prueba requerida frente a la Unión, de entidad autárquica o de empresa pública federal si, en la localidad, no hubiese juzgado federal. $\$ 5^{\circ}$. Se aplica lo dispuesto en esta Sección a aquel que pretenda justificar la existencia de algún hecho o relación jurídica para la simple documentación y registro, y sin carácter contencioso, quien expondrá su intención en un petición fundamentada 
como el professor Eduardo Talamini expressa: "Antes e independentemente del processo, por uma serie de razones: al momento de evaluar las possibilidades efectivas em uma futura y eventual disputa litigiosa, se puede estimar los costos de tal disputa, verificar las possibilidades de los términos de um posible acuerdo com el adversário y asi por delante.". (Talamini, 2016, p.2). (Traduccion Libre)

En este orden de ideas tenemos que recordar que el código trae consigo la premisa orientada al comprender que el único destinatario de la prueba no es solamente el convencimiento del juez, sino también el convencimiento para las partes, con la finalidad de llegar acuerdos que eviten una futura acción en un proceso principal (Sampaio, 2019, p.118), que además de arrojar criterios de pacificación de conflictos bajo la idea de economía procesal y eficiencia, solamente llenaría más el poder judicial y dilataría los plazos razonables de una sentencia inmutable.

El siguiente aspecto a analizar como se viene desarrollando el presente trabajo es numeral siguiente, basado en la función de evitar o justificar una futura acción, es decir en una acción de un proceso principal, y por último el artículo 381 numeral $3 \mathrm{del} \mathrm{CPC/2015,}$ viene a confirmar lo expuesto anteriormente, es decir centra su objeto en brindar a las partes los elementos necesarios para determinar la necesidad o no de una acción futura en un juicio principal además de orientarse a formar las posibilidades de una autocomposición para ser luego idealmente homologada por el tribunal. Es evidente que la acción autónoma de producción anticipada de la prueba puede realizarse con todos los medios probatorios, sean típicos o atípicos esto en consonancia a lo que reza el artículo $369 \mathrm{del} \mathrm{CPC} / 2015^{10}$.

Otra innovación que quiero plantear, es el aspecto de como este código entiende la competencia para esta figura jurídica, ya que expone la competencia para practicar la prueba anticipada será en donde la prueba misma deba de ser producida o en su defecto, en el domicilio del demandado esto como vía excepcional, es decir en el caso de prueba anticipada de una prueba testifical que la persona viva en casa de la otra parte (Didier Jr, 2019, p. 195).

El CPC/2015 es claro al no ligar una conexión de competencia frente al tribunal que en su respectivo caso debería de conocer de la acción principal, en el caso de que este posiblemente existiese, como ocurre en otros ordenamientos jurídicos o más específicamente como en el código de proceso civil 2007 de Honduras.

Asimismo quiero enfatizar en el tipo de sentencia que este proceso tiene, así con los criterios que se adoptan en base a la defensa del mismo, Para esto el artículo 382 del CPC/2015 plantea los criterios relacionado a ello. ${ }^{11}$

10 Art. $369^{\circ}$. Las partes tienen el derecho de emplear todos los medios legales, así como los moralmente legítimos, aunque no estén especificados en este Código, para probar la verdad de los hechos en que se basa en pedido o la defensa, e influir eficazmente en la convicción del juez.

11 Art. $382^{\circ}$. En la petición, el requirente presentará las razones que justifican la necesidad de anticipación de prueba y mencionará con precisión los hechos sobre los cuales la prueba recaerá. $\S 1^{\circ}$. El juez ordenará, de oficio o a requerimiento de parte, la citación de los interesados en la producción de la prueba o en el hecho a ser probado, salvo si fuese inexistente el carácter contencioso. $\$ 2^{\circ}$. El juez no se pronunciará sobre la ocurrencia o no del hecho ni sobre las respectivas consecuencias 
Tal y como reza este artículo en su numeral dos, el juez nunca se pronunciara sobre ningún hecho y sobre ninguna consecuencia jurídica en la sentencia, es decir sobre los aspectos de posible derecho material afectado, dando por finalizado el proceso, dejando clara la posición del juez como organizador de las normas instructoras para garantizarse el derecho a la prueba, en este caso la acción de producción de prueba anticipada, al no ser de carácter contencioso, pero no con la lógica de la jurisdicción voluntaria, a pesar de esto, no significa que no se pueda hacer una tutela provisoria en el caso de urgencia de la prueba producirse (Didier JR, 2019, p.195). bajo las reglas contempladas en el CPC/2015 por ello cabe destacar que este criterio en sentido estricto si cabria recurso de agravio según la legislación brasileña, al tenor que puede existir la posibilidad que el juez se adelante a la decisión del mérito o sobre el contenido mismo de la prueba (Yarshell, 2015,p.1043) por lo que la idea en cuanto la posición de la defensa del reo o demandado, simplemente tiene la posibilidad de presentar defensa en cuanto a la competencia del órgano jurisdiccional o jurisdicción, o cuestiones de forma, mas no puede pronunciarse bajo alguna cuestión de fondo recordando que la prueba anticipada está orientada a las hipótesis ya expresadas.

jurídicas. $\S 3^{\circ}$. Los interesados podrán requerir la producción de cualquier prueba en el mismo procedimiento, siempre que esté relacionada al mismo hecho, salvo si su producción conjunta acarrease excesiva demora. $\$ 4^{\circ}$. En este procedimiento, no se admitirá defensa o recurso, salvo contra la decisión que rechace totalmente la producción de la prueba discutida por el requirente originario

\section{PRUEBA ANTICIPADA EN EL DERECHO PROCESAL VIGENTE DE HONDURAS}

Hasta este punto hemos abordado figuras jurídicas que pueden ofrecer las herramientas para que las partes a través de la información pre proceso, puedan llegar a solucionar sus conflictos a través de acuerdos extrajudiciales, por esta razón ha llegado el momento de plantear bajo la realidad de Honduras que institutos jurídicos podríamos utilizar para esta función o más específicamente la prueba anticipada podría o no tener esta función en el derecho hondureño.

El Código procesal Civil del año 2007 212 , contempla en su estructura, específicamente en el Libro Segundo denominado "Las Pruebas", Titulo primero, capítulo VI, en sus artículos 246 hasta el 250, lo concerniente a la prueba anticipada y el aseguramiento de la prueba. Esta división es fundamental, ya que el legislador pretende que al ser regulados en la estructura supra citada, enfatiza en la diferencia entre estos institutos, que es la prueba anticipada propiamente dicha, y el aseguramiento de prueba ${ }^{14}$ y no en un rubro cautelar específicamente si no en un una mescla entre cautelar y materia probatoria.
12 En adelante CPC/2007.

13 Todos los Artículos jurídicos que se citen de la Legislación Procesal Hondureña del 2007 serán de la obra Proyecto fortalecimiento del poder judicial de Honduras cooperación española código procesal civil comentado honduras.coordinador de la obra magistrado Juan Miguel Carreras Maraña, Tegucigalpa: Editorial, 2009.

14 Asimismo estos conceptos de Prueba Anticipada y Aseguramiento de Prueba, en ningún caso debe de confundirse ni entre ellas ni con las figuras de las medidas cautelares y las diligencias previas propiamente dichas. 
Es por esto que el artículo $246^{15}$ tipifica los supuestos de anticipación de la prueba.

Lo primero que nos percatamos en este artículo es que contempla un espacio-tiempo para iniciar una prueba anticipada sea antes de un proceso principal o en el caso de que ya se encuentre iniciado este, se podrá producir antes de la práctica de las pruebas es decir de la audiencia probatoria, sin embargo por la pertinencia del presente artículo analizaremos la Prueba anticipada antes de iniciar un proceso.

Al Solicitar una prueba anticipada al poder judicial antes de iniciar un proceso, se deberá como presupuesto obligatorio demostrar que existe una urgencia o peligro, para producir la prueba o para salvaguardar la fuente probatoria, además de esto se deberá presentar ante el tribunal que sea competente para conocer el futuro proceso al cual estará ligada esa prueba anticipada para su eficacia.

Asimismo tal y como reza el articulo $247^{16}$

15 ARTICULO 246.- SUPUESTOS DE ANTICIPACION DE LA PRUEBA 1. Antes de iniciar en cualquier proceso, el futuro demandante podrá solicitar la práctica anticipada de algún acto de prueba, cuando se dieran razones de urgencia o existiera temor fundado de que, por causa de las personas o por el estado de las cosas, dichos actos no puedan realizarse en el momento procesal ordinario. La solicitud se dirigirá al juez o tribunal que se considere competente para el conocimiento de la pretensión principal, que examinará de oficio su propia jurisdicción y competencia. Durante la tramitación del proceso cualquiera de las partes podrá solicitar la práctica anticipada de prueba en los casos a que se refiere el numeral anterior

16 ARTÍCULO 247.- PROPOSICION Y PRÁCTICA DE PRUEBA ANTICIPADA AL INICIO DEL PROCESO. 1. La proposición de prueba anticipada se hará conforme a lo dispuesto en este Código para cada medio probatorio, exponiendo las razones en que se apoye la petición. Asimismo el solicitante designará la persona o personas a las que se proponga demandar, que serán citadas, con al menos cinco (5) días de antelación, para que puedan intervenir en la práctica la prueba anticipada se deberá establecer como polo pasivo el posible futuro demandado o futuros demandados, asimismo el numeral tercero establece una imposición dela validez de la prueba anticipada, dándole su eficacia probatoria solamente si se presente la acción principal dentro de un plazo de un mes por regla general, con estos puntos podemos percatarnos que la naturaleza de la prueba anticipada regulada por el CPC/2007 no es más que proteger la fuente probatoria como la producción de la misma a través de un medio probatorio basado exclusivamente en el presupuesto de la urgencia, ahora bien surge la pregunta ¿podría un ciudadano hondureño solicitar una prueba anticipada para efectos de información y por tanto para buscar una conciliación o verificar si existe posibilidad o no de ganar potencial proceso futuro?

En un primer escenario vemos que la norma jurídica procesal hondureña limita esas atribuciones, estableciendo un plazo obligatorio, planteando una dependencia a un proceso futuro, y más todavía obligando la competencia del tribunal para conocer tanto del proceso futuro como de la prueba anticipada, por lo tanto la respuesta en este sentido es negativa.

del medio de prueba. Si el juzgado o tribunal estimare fundada la petición, accederá a ella, disponiendo su práctica en la forma ordinaria. 2.No se otorgará valor probatorio a lo actuado si la demanda no se interpusiere en el plazo de un mes desde que la prueba anticipada se practicó, salvo que se acreditare que, por fuerza mayor o caso fortuito, no pudo iniciarse el proceso dentro de dicho plazo. 3. Para los efectos de la inmediación la prueba anticipada será introducida en la audiencia probatoria mediante su lectura y de ser posible su reproducción. $4 \mathrm{La}$ prueba practicada anticipadamente podrá realizarse de nuevo si, en el momento procesal oportuno, fuera posible llevarla a cabo y alguna de las partes así lo solicitara. En tal caso, el tribunal ordenará su práctica y valorará según las reglas de la sana crítica tanto la realizada anticipadamente como la efectuada en el momento procesal oportuno. 
Es en este punto donde podemos ver que la misma legislación procesal limita los chances de ofrecer salidas auto compositivas a algunos conflictos dentro de la sociedad, por ello es que deseo plantear que la prueba anticipada regulada por el CPC/2007 perfectamente puede aplicarse como en la realidad jurídica brasileña principalmente por las consideraciones siguientes: Primero El profesor Flavio Yarshell ${ }^{17}$ expresa dos criterios fundamentales para comprender el derecho a prueba, consistentes principalmente en distinguir el que es Derecho a Prueba y lo que es Derecho de Probar, El derecho de probar es básicamente todo lo abordado hasta este momento, es decir, una garantía constitucional sustentada por el derecho de acción y de la defensa que está orientada precisamente a las partes ya que el Derecho de probar para su materialización es a instancia de parte haciendo uso de los medios probatorios típicos o atípicos moral y lícitos que puedan usarse dentro de un proceso judicial, y destinada al convencimiento del juez en donde su eficacia y eficiencia consiste en garantizar la admisión, la participación, la producción y la valoración de la prueba, al menos esta es la definición clásica que se venía adoptando (Yarshell, 2009, p.209).

Al Referirme a la eficacia, es evidente que estoy focalizándome en una eficacia probatoria, misma que tiene una serie de escenarios para su cumplimiento, De esta forma FERRERIA (2018) denomina la eficiencia probatoria como "[...] Eficiencia en

17 Profesor Brasileño, autor de la obra Prova Antecipada sem o requisito da Urgencia Direito Autónomo a Prova del año 2009, que fue objeto de su disertación de Doctorado, es uno de los mayores influyentes sobre la temática en toda la doctrina procesal Brasileña. el ámbito probatorio significa la existencia de los medios técnicamente aptos a demostración del ocurrencia o incurrencia de los hechos y para tal objetivo, las técnicas dispuestas sean calibradas conforme a las necesidades que se presentan" (p,579). (Traduccion Libre)

Sin embargo, la idea del derecho a prueba ha ido evolucionando y por tanto superando algunos criterios por la doctrina, al tenor que no sería un error seguir sosteniendo la idea que lo expresado define al derecho de probar pero si sería un error decir que solamente esos criterios denominan o engloban el derecho a prueba, por lo que cada vez las partes juegan un rol más participativo y protagonista en la ciencia procesal y en el proceso judicial como tal, este escenario no se distingue en el ámbito de la prueba judicial, al final y acabo las partes son las que mejor conocen el conflicto y por tanto las pruebas de las alegaciones de los hechos que ellas realizan, en este orden de ideas la prueba no está más destinada solamente al convencimiento del juez, si no que a las partes mismas, como aquellas sujetas de la relación procesal o material en sus respectivos casos, ya que estas pruebas así como podrían ayudar al juez para motivar una sentencia y formar su convicción, tiene una función de fomentar convicción también a las partes ya que la prueba es aquel soporte cognoscitivo para demostrar determinada verisimilitud de las alegaciones de un hecho, que arroja elementos que podrían considerarse información sea para la viabilidad de una acción principal o sea para evitarla así como simplemente para información a las partes (Yarshell, 2009, p.211).

Segundo: El derecho a prueba goza de 
autonomía al efecto de derivarse del derecho de acción, mismo que era definido por Couture (1958) como "El poder jurídico que tiene todo sujeto de derecho, de acudir a los órganos jurisdiccionales para reclamarles la satisfacción de una pretensión." (p.57) de esta forma se encuentra tipificado en el artículo 90 constitucional hondureño, por lo que goza rango de derecho fundamental - constitucional, es en este sentido que el profesor Flavio Luiz Yarshell expresa: [...] El derecho a prueba puede ser entendido, entonces, como derecho simplemente para la obtención de cierta providencia de instrucción, sin la necesaria vinculación directa con el derecho de acción ejercido para el pleito, para la declaración del derecho (o con el ejercicio de la defensa en el proceso instaurado en esos términos) relativamente otorgada a una situación substancial. Bajo este ese prisma, el derecho a prueba gana un cierto sentido de Autonomía. (Yarshell, 2009, p.211). (Traduccion Libre).

Todo esto se relación con las palabras del profesor Pico I Junoy, "El fundamento último de la prueba anticipada no es otro que el de garantizar la mayor virtualidad del derecho a la prueba" (JUNOY, 1996, p.162).

Así como existe un despliegue en base al derecho de acción este como sustentador del principio de la inviabilidad de la jurisdicción (u obligación del poder judicial de conocer esa acción) para tutelar el derecho de probar durante un proceso judicial, también existe el despliegue del derecho de acción para el derecho a prueba, que consiste principalmente en la pre-constitución de una prueba, con una naturaleza de investigación, de forma autónoma a un proceso judicial o a una relación de derecho material o a un plazo determinado para presentar dicha acción (Yarshell, 2009, p.211).

Es por esta razón específicamente que el derecho a prueba es autónomo por su condición de desvinculación de un proceso principal o el reconocimiento de un derecho material o no dentro de ese proceso, ya que el principal objetivo del derecho autónomo a prueba es precisamente el de información o investigación y de obtener la prueba. (Yarshell, 2009, p.211).

En otras palabras el derecho a prueba es un derecho de investigación (Yarshell, 2009, p.213). Esto con la idea que el derecho a prueba otorga la facultad de investigación de información a las partes de manera autónoma a un proceso que deba de resolver o no un proceso en relación a un derecho material en disputa. Por lo que el Derecho a prueba plantea un escenario de estimular la jurisdicción de una nación a través del derecho de acción para la mera obtención de una prueba solo con la naturaleza de información, o investigación a los ciudadanos como ya en varias ocasiones he planteado.

Tercero: Por lo tanto la prueba como ya hemos abordado tiene como destinatario al juez, sin embargo, esta idea es evidentemente superada ya que el juez no solamente es destinatario de la prueba sino que también las partes, y estas partes juegan un rol fundamental en cuanto al derecho a prueba y sobre todos por modelos de cooperación como actores dinámicos dentro de la relación procesal, asimismo este es un derecho que se consagra en la constitución de Honduras, 
reconociendo firmemente la garantía del derecho fundamental a prueba de todo ciudadano, derivado del derecho de acción ( Beltrán, 2016 p. 51).

Al tener las partes la facultad de ser destinatarios de la prueba junto con el juez y tener su derecho de prueba con grado constitucional, el presupuesto de la urgencia tipificada en la legislación infra constitucional procesal, no debería de ser un obstáculo en cuanto a la admisión de dicha acción para requerir un medio probatorio, haciendo uso del poder judicial para mera pre constitución de la prueba o investigación, ya que esta se encuentra regulada en una norma jurídica de rango inferior que está obligada al respeto de la constitución de la republica de Honduras, en virtud que la urgencia no puede en ninguna forma limitar el derecho constitucional fundamental a prueba, por lo que los jueces deben de interpretar la situación inconstitucional de dicho artículo en el caso hondureño no por denominar la urgencia como presupuesto, pero si por denominar la urgencia como único presupuesto de admisión, lo que es por todo lo expuesto una evidente violación al derecho autónomo de prueba consagrado en la constitución como un derecho fundamental inviolable. $\mathrm{O}$ como bien señala Jordi Ferrer Beltrán En este sentido deben considerarse inconstitucionales aquellas limitaciones a la posibilidad de aportar pruebas relevantes impuestas no ya por el juzgador si no legislativamente. En este sentido deben de considerarse inconstitucionales aquellas limitaciones a la posibilidad de aportar pruebas que no resulten justificadas en la protección de otros derechos fundamentales en conflicto (Beltrán, 2016 p. 53).
En este sentido la experiencia brasileña como ejemplo más cercano al Civil Law y más aún la experiencia de derecho Anglosajón, esta acción probatoria perfectamente puede proveer información suficiente para que las partes puedan formar una autocomposición de la Litis, verificar sus chances de ganar o perder un juicio futuro, y así no perder tiempo ni saturar el poder judiciario, haciendo el derecho procesal más eficiente para su finalidad, o como verificación de información, por lo que concluyo que en el derecho hondureño los jueces deben de flexibilizar e iniciar con el rompimiento de esquemas rígidos que lo único que hacen es limitar, burocratizar y perturbar el acceso a la justicia civil.

\section{CONCLUSION}

Hemos analizado brevemente por la naturaleza de este trabajo varias figuras jurídicas que pueden ofrecer información pre proceso en varios países del mundo, con la finalidad que las partes envueltas en el conflicto perciban cuáles son sus posiciones y puedan tanto formar una autocomposición del conflicto o verificar si existe viabilidad o no para iniciar un proceso futuro.

La legislación nacional debe siempre apoyar y fomentar la conciliación o métodos alternos de solución de conflictos, ya que esto como establecí al inicio estamos bajo una clara cultura litigiosa, por lo que este problema no solamente abarca a la legislación pero, no podemos negar que la misma legislación esta subsumida en este panorama, lo expuesto hasta aquí solo es un ejemplo de muchos. 
Por lo que este cambio debe de iniciar desde las reformas legislativas además de establecer en el pensum académico criterios adecuados para ir rompiendo con esta cultura, principalmente en la abogacía, para que esta a través del tiempo se va traduciendo a las personas de la sociedad.

Asimismo la Prueba anticipada solamente es una figura jurídica de las muchas que pueden tener este rol de información para las partes para que puedan solventar sus conflictos, ya que existen otras como el Habeas Data, Diligencias Preliminares, Centros Conciliación etc. Que evidentemente deben de ser estudiados con mucho mayor rigor.

El mundo ya percibió que el conflicto no se acaba ni se acabara y que por tanto el poder judicial no puede ser la única puerta de solución misma porque no es ni la mejor solución para todos los casos y porque no tiene capacidad para atender todos los casos, por lo que buscar nuevos horizontes será el trabajo y desafío arduo tanto de la academia como de los gobiernos y la sociedad.

\section{BIBLIOGRAFIA}

BENTAM, J. (1971) Tratado de las pruebas judiciales. Trad. Traite des preuves judiciareis por Manuel O. F. Buenos Aires. EJEA. v. 3.

CAVANI, R. (2015) CPC brasileiro traduzido para a língua espanhola: código de proceso civil brasileño de 2015 BRASIL. Ley $\mathrm{n}^{\circ}$ 13.105, de 16 de Março de 2015. Coord. ARRUDA, Teresa Alvim. DIDIER JUNIOR, Fredie. Tradução CAVANI,
RENZO. Salvador. Jus Pudivm.

Chase, O. G. (2014). Direito, cultura e ritual. Sao Paulo. Marcial Pons.

Laux, F. D. M. (2015). Relações entre a Antecipação Da Prova Sem O Requisito Da Urgência e a Construção de soluções autocompositivas. Revista de Processo, v. 242, p. 1-19.

Corte Suprema de Justicia de Honduras. (2006). Plan Nacional De Erradicación De La Mora Judicial. Disponible en : http://www.poderjudicial.gob.hn/PNEMJ/ Paginas/default.aspx. Ultimo acceso: 30/8/2020

COUTURE, E. J. (1958). Fundamentos del Derecho Procesal Civil. Buenos Aires. Roque De Palma.

DIDIER JR, F. (2019) Produção antecipada de provas: Aspetos Controvertidos. En SAMPAIO, B. A. F. RODRIGUES, D. C. ANTUNES, T. C. Produção Antecipada da Prova- questões relevantes e aspectos polêmicos. (p.118 -119). Londrina,PR. Thoth.

FERREIRA, W.S. Transições paradigmáticas, máxima eficiência e técnicas executivas típicas e atípicas no Direito Probatório. En DIDIER J. F. Direito Probatório. Salvador Bahía. Jus Podivim.

JUNOY, J. P. I. (1996). El derecho a la prueba en el proceso civil. Barcelona. José María Bosch Editor. 
NERY JUNIOR, N. ANDRADE, R. M. N. (2018). Código de Processo Civil Comentado, São Paulo, Thompson Reuters Brasil Revista Dos Tribunais. 17 ed.

Proyecto Fortalecimiento Del Poder Judicial De Honduras Cooperación Española. (2009). código Procesal Civil Comentado Honduras. coordinador de lá obra magistrado Juan Miguel Carreras Maraña, Tegucigalpa Editorial.

SAMPAIO, B. A. F. (2019). A produção Antecipada da prova: Aspectos Gerais e Natureza da Sentença. En SAMPAIO, B. A. F. RODRIGUES, D. C. ANTUNES, T. C. Produção Antecipada da Prova- questões relevantes e aspectos polêmicos. (p.118 -119). Londrina,PR. Thoth.

SCHNEIDER, S. SCHIMITT, C. J. (1998). O uso do método comparativo nas Ciências Sociais. Cadernos de Sociologia. Porto Alegre, 1998. V.9 p.49-87. Disponible en: https://www.academia.edu/5815441/O uso do $\mathrm{m} \% \mathrm{C} 3 \% \mathrm{~A} 9$ todo comparativo nas ci $\% \mathrm{C} 3 \% \mathrm{AAncias}$ so ciais. Último acceso: 15 Feb. 2020.

Silveira, S. A. (2010). Acordos Incentivados Uma Contribuição Britânica Nos Caminhos Buscados Pelo Judiciário Brasileiro. Universidade De Sao Paulo.

TALAMINI, E. (Octubre 2016). Produção Antecipada de Prova No Código De Processo Civil De 2015. Revista dos Tribunais. Nro. vol. 260, p. $75-101$.

YARSHELL, F. (2016). Breves comentários ao novo código de processo civil. En WAMBIER, Teresa A. DIDIER JR, F., TALAMINI E. DANTAS, B. São Paulo. Revista dos tribunais. 2 eds.

YARSHELL, F. L. (2009). Antecipação da prova sem o requisito da urgência e direito autônomo à prova. São Paulo. Malheiros. 